\title{
Analyst
}

\section{FTIR microscopy of biological cells and tissue: data analysis using resonant Mie scattering (RMieS) EMSC algorithm}

\author{
Paul Bassan, $\dagger^{a}$ Ashwin Sachdeva, $\dagger^{a}$ Achim Kohler, ${ }^{b}$ Caryn Hughes, ${ }^{a}$ Alex Henderson, ${ }^{a}$ Jonathan Boyle, ${ }^{c}$ \\ Jonathan H. Shanks, ${ }^{d}$ Michael Brown, ${ }^{e}$ Noel W. Clarke ${ }^{e}$ and Peter Gardner ${ }^{* a}$
}

\author{
Received 10th November 2011, Accepted 16th January 2012 \\ DOI: 10.1039/c2an16088a
}

\begin{abstract}
Transmission and transflection infrared microscopy of biological cells and tissue suffer from significant baseline distortions due to scattering effects, predominantly resonant Mie scattering (RMieS). This scattering can also distort peak shapes and apparent peak positions making interpretation difficult and often unreliable. A correction algorithm, the resonant Mie scattering extended multiplicative signal correction (RMieS-EMSC), has been developed that can be used to remove these distortions. The correction algorithm has two key user defined parameters that influence the accuracy of the correction. The first is the number of iterations used to obtain the best outcome. The second is the choice of the initial reference spectrum required for the fitting procedure. The choice of these parameters influences computational time. This is not a major concern when correcting individual spectra or small data sets of a few hundred spectra but becomes much more significant when correcting spectra from infrared images obtained using large focal plane array detectors which may contain tens of thousands of spectra. In this paper we show that, classification of images from tissue can be achieved easily with a few $(<10)$ iterations but a reliable interpretation of the biochemical differences between classes could require more iterations. Regarding the choice of reference spectrum, it is apparent that the more similar it is to the pure absorption spectrum of the sample, the fewer iterations required to obtain an accurate corrected spectrum. Importantly however, we show that using three different non-ideal reference spectra, the same unique correction solution can be obtained.
\end{abstract}

\section{Introduction}

Fourier transform infrared (FTIR) spectroscopy has been used to acquire biochemical information from biomedical samples for a number of years with varying degrees of success, see recent reviews. ${ }^{1-4}$ This variability is largely due to the fact that the samples, normally cells or intact tissue are far from ideal from a spectroscopic point of view. The acquisition of a spectrum that reliably represents the biochemistry of the sample is difficult to

${ }^{a}$ Manchester Interdisciplinary Biocentre, University of Manchester, 131 Princess Street, Manchester, M1 7DN, UK. E-mail: peter.gardner@ manchester.ac.uk; Fax: +0161 306 5201; Tel: +0161 3064463

${ }^{b}$ Nofima Mat, Centre for Biospectroscopy and Data Modelling, Osloveien 1, 1430 As, Norway

${ }^{c}$ Research Applications \& Collaboration Team, IT Services for Research, Devonshire House, The University of Manchester, Manchester, M13 9PL ${ }^{d}$ Department of Histopathology, The Christie NHS Foundation Trust, Manchester, UK, M20 4BX, UK

${ }^{e}$ Genito Urinary Cancer Research Group, School of Cancer and Enabling Sciences, Paterson Institute for Cancer Research, The University of Manchester, Manchester Academic Health Science Centre, and Department of Urology, The Christie NHS Foundation Trust, Manchester, UK, M20 4BX, UK

$\uparrow$ Authors have contributed equally. obtain, since the scattering properties of biomedical specimens, resulting from variations in morphology, lead to severe distortions in the measured spectra. ${ }^{5-8}$ Recently it was shown that the predominant cause of the distortion is due to the phenomenon termed resonant Mie scattering (RMieS) ${ }^{5}$ and to a lesser extent reflections from the sample surface. ${ }^{6}$ Clearly, if the "magnitude" of the distortion due to scattering is greater than the "magnitude" of the biochemical signal difference between samples, then this biochemical information is obscured. In order to solve this problem an RMieS correction algorithm (RMieS-EMSC) was developed that enables an "undistorted" absorption spectrum to be recovered from the measured distorted spectrum ${ }^{9-11}$ and has been successfully used in a number of studies. ${ }^{12,13}$

The RMieS-EMSC algorithm essentially takes a scatter-free reference spectrum $\left(Z_{\text {Ref }}\right)$, and uses this to reconstruct/model the raw spectrum $\left(Z_{\text {Raw }}\right)$ to be corrected. This is done by assuming that $Z_{\text {Raw }}$ is the superposition of $Z_{\text {Ref }}$ and a number of scatter curves; the details of exactly how this is done is documented elsewhere. ${ }^{9,10}$

The correction algorithm is most efficient when the undistorted reference spectrum is close to the desired result. Since the desired result is unknown the user must input a reference spectrum that they think will be a reasonable match. Fortunately, in the case of biological cells and tissue, the basic form of the 
spectra is very similar such that using a "standard" spectrum of, e.g., say Matrigel (a commercial artificial extra cellular matrix that contains all the key components expected in tissue) will give a reasonable result. The consequence of starting with an "arbitrary" reference spectrum, however, is that after a single pass through the algorithm, the corrected spectrum bears similarity to both the reference and the pure absorbance signal from the raw spectrum. ${ }^{9}$ At this point, the spectrum upon visual inspection has much reduced scattering, but it may now contain unwanted spectral characteristics from the reference spectrum. To reduce the contribution of $Z_{\text {Ref }}$, the newly corrected spectrum $\left(Z_{\text {Corr }}\right)$ is subsequently used as the new reference spectrum with the hope that the next correction will result in more accurate representation of the pure absorbance spectrum of interest. Thus, using the above steps, shown schematically in Fig. 1, it is possible to iteratively improve the reference spectrum, such that the algorithm eventually converges upon the pure absorbance spectrum which we are trying to recover. ${ }^{9,10}$

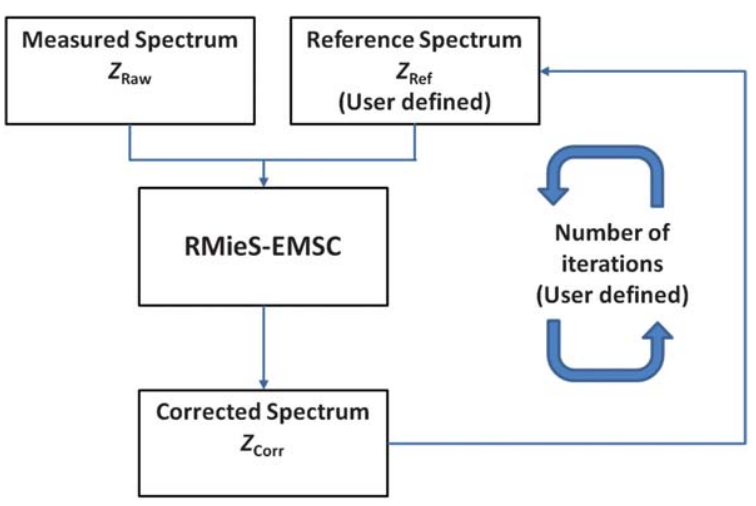

Fig. 1 A simplified schematic diagram of the correction procedure. The user defined inputs are the choice of reference spectrum and the number of iterations used.

Although the algorithm delivers scatter free spectra that appear to represent the intended absorption spectrum it is legitimate to ask how strongly the $Z_{\text {Ref }}$ influences the resultant corrected spectra and how many iterations of the algorithm are required to eliminate this influence or reduce it to a point where it is insignificant.

In this paper we attempt to answer these questions. Our approach is to (i) analyse a simulated data set to see how many iterations RMieS-EMSC algorithm are required to recover the correct classification, (ii) correct a spectrum with three different non-ideal reference spectra and see if the three corrected spectra converge to a unique solution and (iii) investigate the influence of the number iterations on the classification of images consisting of $128 \times 128$ spectra. Analysis of the corrected spectra by comparison to the known result gives further insight into the behaviour of the algorithm.

\section{Materials and methods}

\subsection{Sample preparation}

Paraffin-embedded blocks of prostate tissue samples of patients with benign prostatic hyperplasia $(\mathrm{BPH})$ were obtained following informed consent and ethical approval (Trent Multi-centre Research Ethics Committee 01/4/061). Two tissue sections were cut from each tissue block. One section was fixed on to a low-e MirrIR slide (Kevley Technologies, Ohio, USA) for infrared imaging, and a serial section was fixed onto a glass slide for immunofluorochemical (IFC) staining. Paraffin was removed by washing slides with Citroclear and cold acetone $\left(4{ }^{\circ} \mathrm{C}\right)$, as per a previously published protocol, ${ }^{14}$ and left to dry overnight.

\subsection{Immunofluorochemical staining}

Samples were rehydrated using serial dilutions of ethanol. Heat induced antigen retrieval was performed by immersing the sample in $2.1 \mathrm{~g}$ citric acid (dissolved in sodium hydroxide to attain a $\mathrm{pH}$ of 6.0 ), heating in a microwave to $80^{\circ} \mathrm{C}$ for $20 \mathrm{~min}$, and then allowing to cool to room temperature. Samples were then incubated in $10 \%$ goat serum plus $1 \%$ bovine serum albumin in phosphate buffer solution (PBS) for $30 \mathrm{~min}$ at room temperature. This was followed by an overnight incubation with mouse monoclonal (AE1-AE3) anti-pan-cytokeratin antibody (Thermo Fisher Scientific Inc., Surrey, UK) at $1: 200$ dilution in $1 \%$ bovine serum albumin in PBS in a humidified chamber in the dark. This particular antibody was chosen as it is highly specific in staining epithelial cells in prostate tissue. ${ }^{15}$

Stained sections were then viewed using an Olympus BX51 fluorescence microscope (Olympus America Inc, Melville, NY) via MetaMorph software (Universal Imaging Corporation, West Chester, PA).

\subsection{Infrared imaging}

Serial sections on low-e substrates were interrogated using transflection-mode FTIR imaging spectroscopy on a Varian 670IR spectrometer coupled with a Varian 620-IR imaging microscope (Varian Inc, - now Agilent Technologies, Santa Clara, CA) equipped with a $128 \times 128$ pixel liquid nitrogen-cooled mercurycadmium-telluride (MCT) focal planar array detector. Spectra were collected in the $850-4000 \mathrm{~cm}^{-1}$ range, at a resolution of $4 \mathrm{~cm}^{-1}$, co-adding 64 scans for sample spectra, and 128 scans for the background spectra.

The ATR reference spectra were recorded using a Varian FTS 7000 spectrometer coupled to a Varian 600 UMA FTIR microscope equipped with a germanium ATR accessory. Spectra were recorded in the wavenumber range $4000-850 \mathrm{~cm}^{-1}$ at a spectral resolution of $4 \mathrm{~cm}^{-1}$, and 1 level of zero filling. The background spectrum was recorded with the germanium crystal in air approximately $0.5 \mathrm{~mm}$ above the sample.

\subsection{Spectral pre-processing}

Data analysis was performed using MATLAB Version 2010a (MathWorks Inc, Palo Alto, CA). A noise reduction algorithm based on principal component analysis (PCA) was applied to each image map to improve SNR. The process simply involves performing PCA, then retaining a certain number of PCs (70 in this case) and then reconstructing the original data. The appropriate number of PCs were chosen by examining the residual from the original data matrix minus the reconstructed matrix. When reconstructing the data using 35 PCs, the residual spectra contained only noise and no chemical information, however in 
the interest of retaining as much chemical information and removing as much noise as possible, 70 PCs were used to err on the side of caution. This results in chemical information being preferentially retained in the principal components over the noise which by definition has no underlying pattern. The RMieSEMSC algorithm (version 3) was used to correct the data for Mie scattering effects.

\subsection{Data analysis}

2.5.1 Hierarchical Cluster Analysis (HCA). As an unsupervised clustering tool, HCA was employed to group data into a user defined number of clusters. HCA was implemented using Euclidean distances and Ward's algorithm, ${ }^{16}$ this is commonly used to cluster vibrational spectroscopic data into a defined number of groups without any prior information of class membership. ${ }^{1,17,18}$ Clustering was performed on data after appropriate pre-processing which is stated later for each specific experiment.

2.5.2 Artificial Neural Networks (ANNs). An artificial neural network (ANN) is a method of supervised machine learning and pattern recognition which has been used with great success in vibration spectroscopy classification problems. ${ }^{19}$ In this report, the conjugate gradient back-propagation ANN learning algorithm was used as it was shown to give higher classification accuracy and faster training times than the other available algorithms in the MATLAB Neural Network Toolbox. A three layer ANN was used (input, hidden and output layer) with 20 hidden neurons.

The basic premise of supervised machine learning in the case of vibrational spectroscopy data is to subject the data to spectra with labels assigning the class membership (hence supervised). Then external validation and testing data can be used to check accuracy and optimise until a satisfactory classification method has been constructed.

\subsection{High throughput computing using Condor}

The RMieS-EMSC algorithm is programmed and run within the MATLAB environment. A very common process for algorithm development is to design and test within MATLAB due to ease of use, and then finally program in a faster language such as $\mathrm{C} / \mathrm{C}++$ or FORTRAN. We are still in the developmental stage of the algorithm and have concentrated on developing the underlying science rather than performance, however computation times for correcting our FTIR images from a $128 \times 128$ (16384 pixels) focal plane array (FPA) are significantly longer than sample measurement time. As an example, an FTIR image consisting of 16384 spectra, each of which has between 1400-1600 data points (absorbance values) corrected using 20 iterations takes approximately 8 days on our lab computer which is a Quad Core Xeon (2.8 GHz, $18 \mathrm{~GB}$ memory).

As a solution for the long MATLAB computation times, we have started to use Condor high throughput computing ${ }^{20}$ (HTC) using a Condor pool at the University of Manchester. This Condor pool consists of over 1400 MATLAB enabled 64-bit Linux desktop computers which can be given jobs to complete during times when the computer is not used, such as during lunch times, evenings and weekends. The benefit of using a Condor pool for us is that we are able to harness a huge amount of computing power at very little cost. By splitting our 16384 spectra FTIR images into approximately 1000-1500 job packets, we can send out the jobs to free computers. During evenings, we can now correct an FTIR image using the same settings as previously mentioned in less than 20 minutes, a significant saving over 8 days.

We recognise that more efficient programming (both language and functions) is the ideal first step towards algorithm optimisation, however for minimal time investment and zero cost we have found a method for correcting our images in a more feasible time frame allowing us to work with RMieS corrected FTIR images and large single cell data sets.

\section{Results and discussion}

\subsection{Number of iterations of the RMieS-EMSC algorithm}

As previously reported, a spectrum that has been corrected with just a single pass through the RMieS-EMSC algorithm will have most of the scattering removed but will contain some information from the reference spectrum as well as the sample., ${ }^{9,10}$ This problem is reduced by using this corrected spectrum as the new reference spectrum. Note that it is the original raw data $Z_{\text {Raw }}$ that is corrected during each iteration thus using this iterative process the influence of the original reference spectrum is continually diluted to a point where all the key chemical features come from the measured sample spectrum. The question of course is how many iterations are required before the influence of the reference spectrum has been diluted to a point where it is inconsequential? The answer very much depends on the experiment being performed.

A common theme of FTIR biomedical research is to develop rapid cost effective screening methods where the aim is to distinguish rapidly between classes of samples $e . g$. healthy cells versus cancerous cell or cells treated with drug A compared with those treated with $\mathrm{B}, \mathrm{C}$ and $\mathrm{D}$ etc. In these situations, where rapid classification is more important than detailed spectral interpretation, the quality of the corrected spectra may not need to be perfect, just good enough to allow accurate discrimination between the groups. Thus as long as the RMieS distortion can be removed to a point where the biochemical differences between classes are greater than the scattering differences this will be sufficient for correct classification based on biochemistry.

To demonstrate this point, four groups of infrared spectra consisting of 100 spectra in each group have been simulated, as per previous work. ${ }^{9,10}$ Fig. 2(a) shows the simulated spectra in the region generally considered of most diagnostic interest. ${ }^{21}$ Fig. 2(b) shows the corresponding PCA scores plot for the simulated data. As can be seen, the data form four distinct clusters. However, to simulate a realistic situation of spectra from four closely related cell lines, the data has been constructed such that clusters have some degree of overlap in PCA score space. To each spectrum, a vector of random numbers was added between the value of -0.015 to 0.015 with a mean of zero. Fig. 2(c) shows the spectra having been artificially distorted by RMieS scattering. This has been achieved by calculating the refractive index and then using this to produce $Q_{\text {ext }}$ curves using 

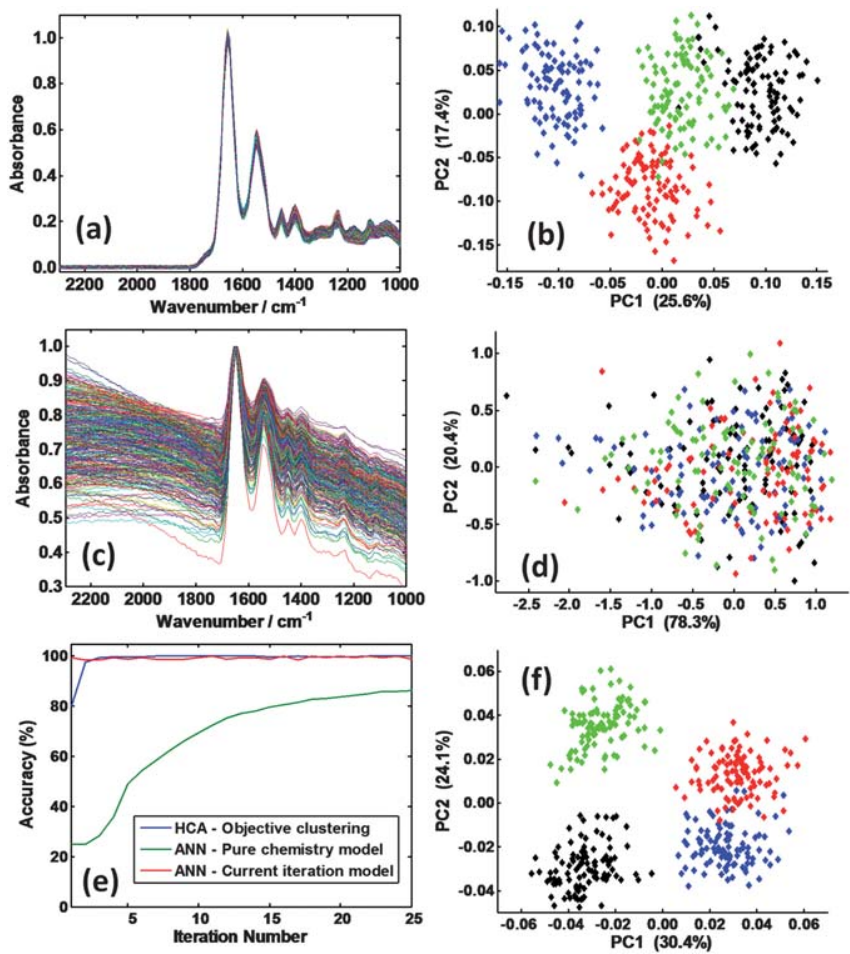

Fig. 2 (a) Four groups of simulated infrared spectra consisting of 100 spectra in each group. (b) PCA scores plot of the data shown in (a). (c) The spectra from (a) incorporating the influence of scattering. (d) The PCA scores plot of the scattered spectra in (c). (e) Classification accuracy as a function of the number of iterations of the RMieS-EMSC algorithm for three different classification models, HCA objective clustering (blue curve), ANN-1, the pure chemistry model (green curve) and ANN-2, the current iteration model (red curve). (f) The PCA scores plot for the data in (c) after 25 iterations of the RMieS-EMSC algorithm.

the van de Hulst Mie scattering approximation..$^{5,22}$ Ten scattered curves were calculated using 10 random particle spheres of radii between $2 \mu \mathrm{m}$ and $7 \mu \mathrm{m}$ and added with a random weighting to the spectra in Fig. 2(a). The resulting PCA scores plots of the scattered spectra Fig. 2(d) show that separation and classification cannot be achieved with this data. The spectra have subsequently been treated with the RMieS-EMSC correction algorithm, using a spectrum of Matrigel as the first guess/reference spectrum. After each iteration, $n$, the data are analysed a number of different ways, the first uses hierarchical cluster analysis (HCA) using Euclidean distances and linkage via Wards algorithm. HCA has proved to be an invaluable tool for the analysis of large spectroscopic data sets and, after PCA, is probably the most commonly used method to investigate patterns in data. ${ }^{17-19}$ The data is clustered into 4 groups using HCA, and the results compared to the known correct assignments. The second and third methods both use artificial neural networks (ANN), which is a pattern recognition tool. ${ }^{19,23}$

ANN-1 uses the undistorted simulated data to build (train) a classification model which remains the same for all iterations, whereas ANN-2 uses the data from iteration $n$ to build the model. Half of the spectra in each class (50 per class) are used for training leaving the remaining available to validate the model (50 per class). Fig. 2(e) shows the accuracy of the classification as a function of the number of iterations $n$ of the RMieS-EMSC algorithm. As can be seen, after just one iteration the HCA model can classify the spectra with $80 \%$ accuracy. Of the ANN models, ANN-1 performs poorly but ANN-2 achieves near perfect classification $99.5 \%$. By the third iteration the HCA model also reaches near perfect cluster assignments. The ANN-1, however, although improves with increasing iterations, starts to plateau after 25 or so. Fig. 2(f) shows the PCA scores plot after 25 iterations of the RMieS-EMSC algorithm and it can seen that the data separates into four distinct clusters, it should be noted that the colour code has been kept constant with Fig. 2(b). The scores plot is different to Fig. 2(b) indicating that the data is not identical as is already known. The loadings of the PCA from each data set that produced Fig. 2(b) and Fig. 2(f) are complicated to understand and make it difficult to draw conclusions regarding what the discriminating features are. The results only from the first 25 iterations have been shown as there was no further significant improvement in spectral quality after this number.

\subsection{Classifications of images}

Infrared spectroscopy has previously been applied in the study of tissue biopsies for the diagnosis of disease, for example prostate cancer. ${ }^{24-26}$ Accurate classification of samples into cancer and non-cancer groups is essential in this research. High quality spectral data is, therefore, a necessity. A number of recent papers have applied the RMieS-EMSC algorithm to correct infrared images to improve classification accuracy. ${ }^{27,28}$ However, the application of the algorithm to infrared imaging has not been objectively evaluated.

Fig. 3(a) and (c) show total absorbance (area under each spectrum) FTIR images of prostate tissue from patient 1 and 2 respectively; the colour scheme is heatmap style such that white indicates high absorbance. Fig. 3(b) and (d) show fluorescence
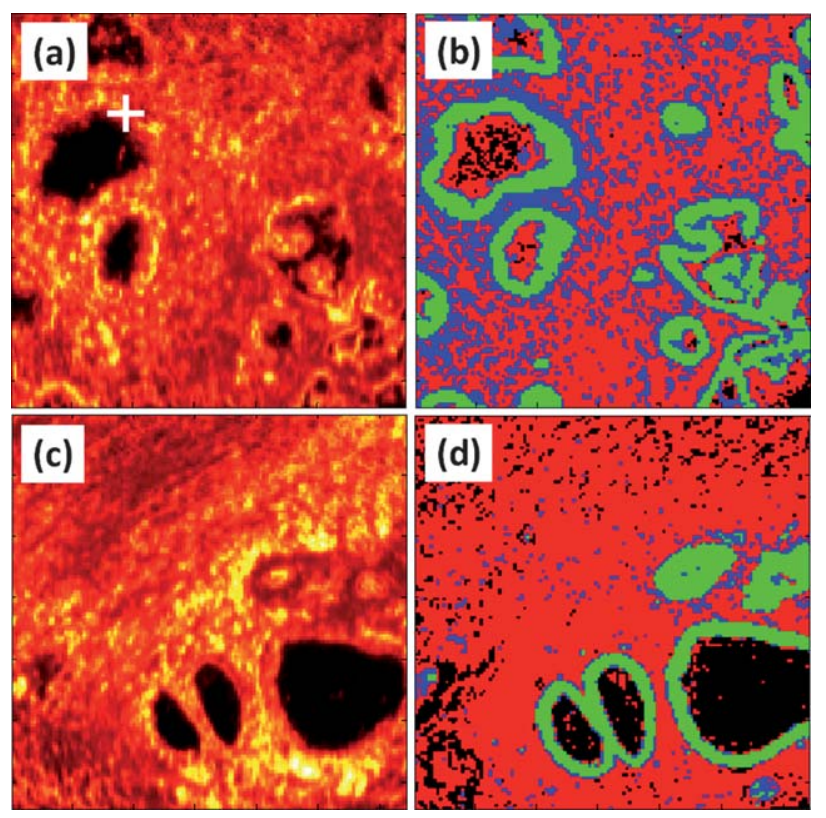

Fig. 3 (a) Total absorbance heatmap FTIR image of prostate tissue from patient 1. (b) Adjacent serial section to (a) which has been stained and thresholded. (c) and (d) are the FTIR and thresholded staining images for patient 2 respectively. 
images of prostate tissue from the adjacent serial section to Fig. 3(a) and (c) respectively, which have been stained with anti pan cytokeratin antibody which stains for prostate epithelial cells. The green pixels in the images are highly stained and can be assigned unequivocally to epithelial cells. The red pixels represent stroma (low level of staining) and the blue pixels are regions where the level of staining was such that the pixels could not be unequivocally assigned to epithelium or stroma. The black pixels represent areas where essentially no fluorescence intensity (ambient light) is observed indicative of areas where there is no tissue. Quality testing of the FTIR image allows spectra from regions of no tissue to be removed from any analysis.

Fig. 3 demonstrates that the thresholding method of the staining Fig. 3(b) and (d) correlate well with the corresponding FTIR image Fig. 3(a) and (c), illustrating that the staining assignments can be translated to FTIR pixels of the adjacent serial sections.

Compared to the spectral distortion of infrared spectra from single cells, spectra from microtomed tissue sections have been considered to be generally less affected. ${ }^{9,29}$ The surface of tissue sections does not exhibit the same morphological characteristics as isolated single cells hence less scattering. Fig. 4(a) shows the raw spectra from the point marked with a white cross in Fig. 3(a). The tissue spectrum at this point, close to the edge of the lumen,
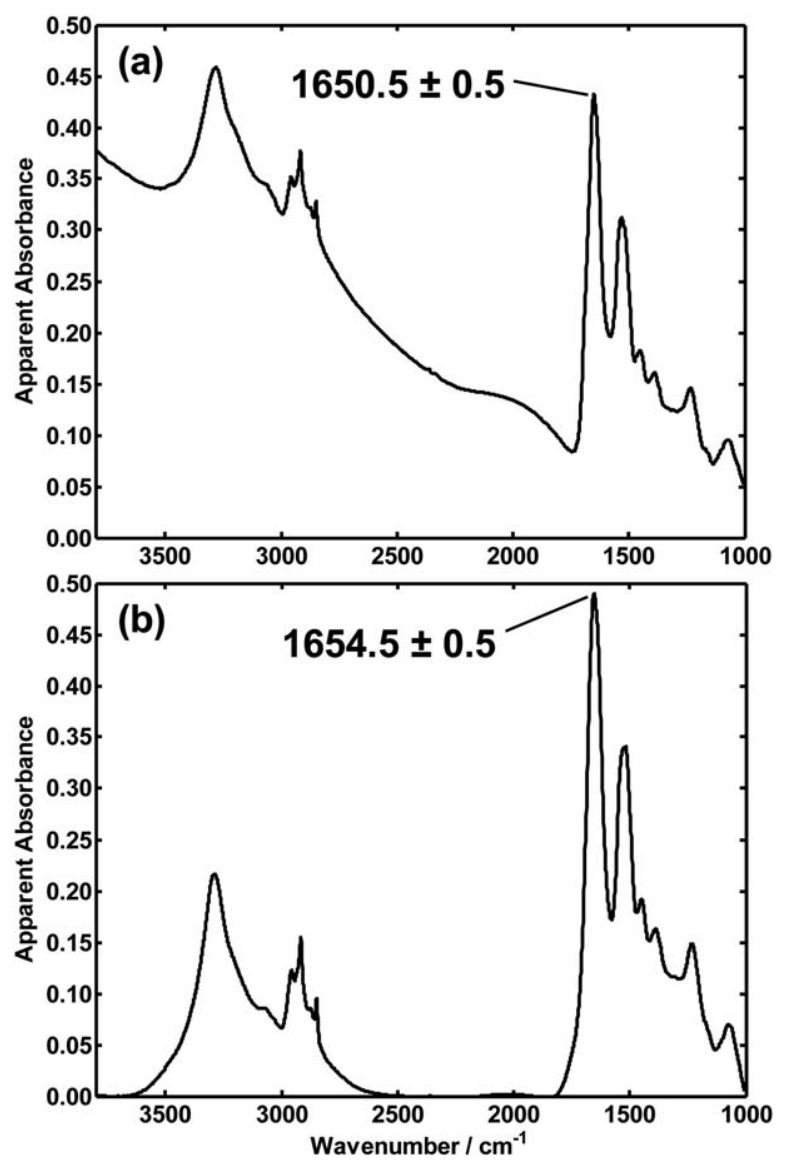

Fig. 4 (a) Raw spectrum from an FTIR image of prostate tissue located at an edge near the lumen of a gland indicated by the white cross in fig $3 \mathrm{a}$ (b) The same spectrum corrected using the RMieS-EMSC after 25 iterations. Data spacing of both spectra was $1 \mathrm{~cm}^{-1}$. shows a strong sloping baseline and a more pronounced decrease in intensity on the high wavenumber side of the amide I band at $1650.5 \mathrm{~cm}^{-1}$. These distortions are classic indications of Mie-type scattering as observed for single cells. ${ }^{5,29}$ Fig. 4(b) shows the same spectrum having been corrected using the RMieS-EMSC correction algorithm (25 iterations). In addition to recovering a flat baseline the RMieS-EMSC correction has shifted the peak position of the amide I band to $1654.5 \mathrm{~cm}^{-1}$. This is significant since peak position of the amide I band can play a major role in classification.

The influence of the number of iterations of the EMSC algorithm on classification of spectra in the infrared image is demonstrated in Fig. 5. The classification has been carried out using ANN. The ANN was trained using data from patient 1 shown in Fig. 3(a). The fluorescence image (patient 1) was overlaid with the corresponding FTIR image (patient1) so that spectra that associated with green pixels in the fluorescence image could be assigned to epithelial cells and those associated with red pixels assigned to stroma. Fig. 5 shows the classification of tissue from patient 2 using the ANN trained on patient 1 . The green pixels again represent spectra classified as epithelium, red pixels represent stroma. After 5 iterations Fig. 5(a) the classification is in reasonable agreement with the stained image Fig. 3(c) however significant improvement is obtained by 10 iterations. In keeping with the observation from Fig. 2(e) there is a law of diminishing return and by 30 iterations Fig. 5(d) there is little further change in the classification of pixels in the image.

\subsection{The influence of the reference spectrum}

We have established in the previous section that classification of the spectra can be achieved after a relatively small number of
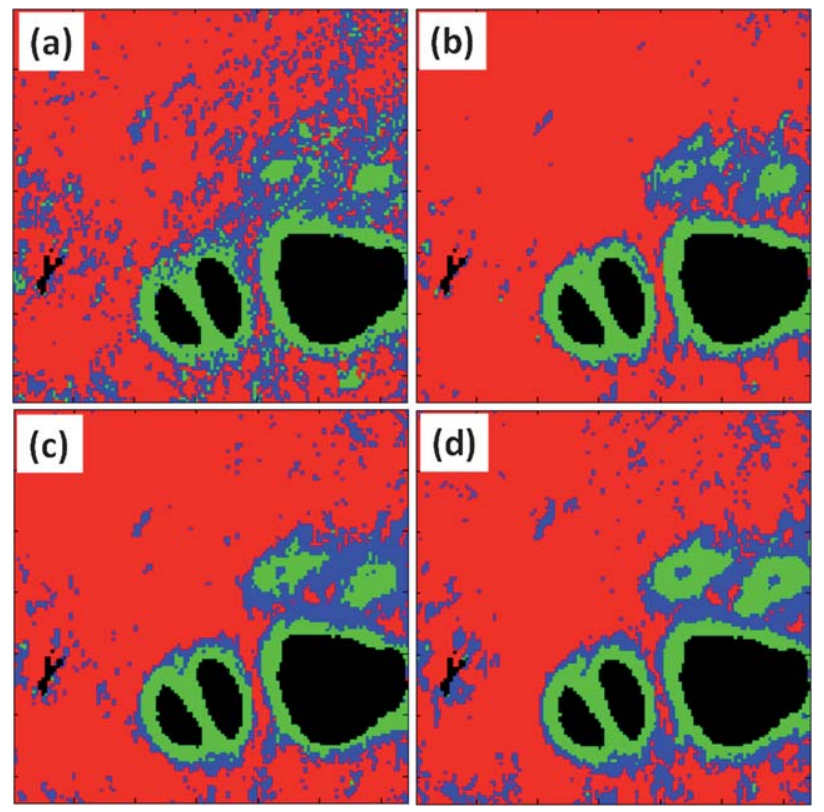

Fig. 5 The classification of patient 2 using patient 1 as the training data, after (a) 5, (b) 10 (c) 20 and (d) 30 iterations of the RMieS-EMSC algorithm. Training data always of same number of iterations as test data. Classification algorithm used was an ANN trained on the FTIR imaging data from Fig. 3(a). 
iterations of the RMieS-EMSC algorithm. Thus for most situations including classification of images this is a computationally manageable task. In cases where fine details of spectral interpretation are essential it may be necessary to increase the number of iterations.

A key question here is whether the starting reference spectrum influences the result of the final correction. In order to examine this, a spectrum from an image of prostate tissue has been corrected using three different reference spectra. The three biological reference spectra that used are (i) transmission spectrum of a thin film of Matrigel, (ii) an ATR spectrum of a different area of prostate tissue, and (iii) an ATR spectrum of a monolayer of renal cells. The ATR spectra were corrected for the wavenumber dependence of the evanescent wave penetration depth so that they are reasonably compatible with an equivalent transmission spectrum. ATR spectra make reasonable reference spectra since they are not influenced to the same extent by RMieS and thus tend to have flat baselines.

Fig. 7 shows the same sample spectrum corrected once using the three different reference spectra. As can be seen the

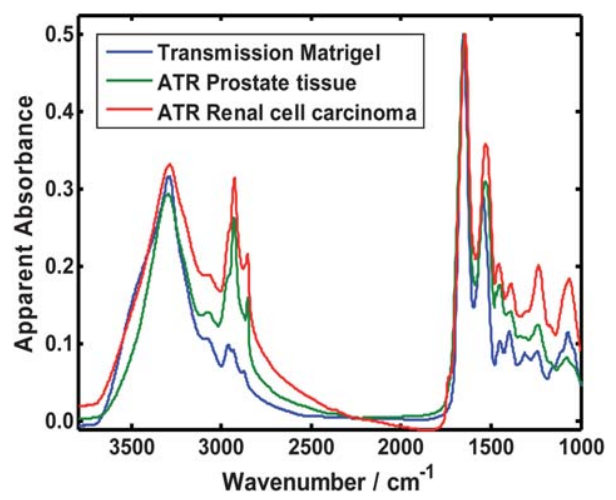

Fig. 6 Three different FTIR spectra of biological samples used as reference spectra: blue curve a transmission FTIR spectrum of Matigel; green curve an ATR FTIR spectrum of prostate tissue; red curve, an ATR FTIR spectrum of a monolayer of renal carcinoma cells.
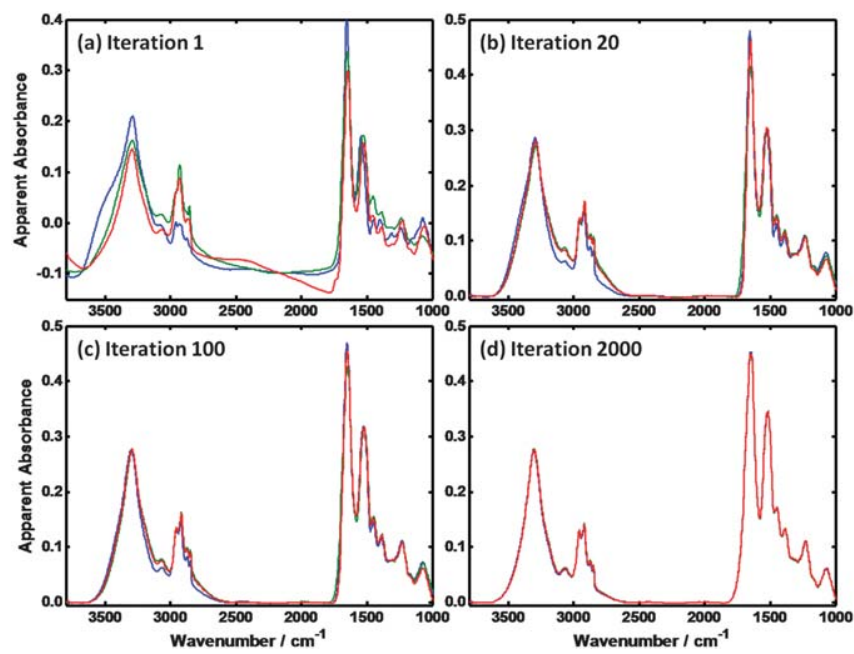

Fig. 7 A single FTIR spectrum of prostate tissue corrected with the three different reference spectra shown in fig 3, after (a) 1, (b) 20, (c) 100 and (d) 2000 iterations of the RMieS-EMSC algorithm. scattering distortion has significantly diminished but has not been eliminated completely. It is clear, however, that the band intensities are different due to the differences in the reference spectra. After 20 iterations Fig. 7(b) the spectra are essentially scatter free and the band positions and intensities are very similar. This is consistent with the data in the previous section that shows that after 20-30 iterations the spectrum is corrected sufficiently such that it is diagnostically useful. The main point of discrepancy is the baseline underneath the $\mathrm{CH}_{2} / \mathrm{CH}_{3}$ stretching region which is not of major diagnostic significance. For completeness the same spectrum has been corrected using up to 2000 iterations. The results at 100 and 2000 are shown in Fig. 7(c) and (d). By 2000 iterations the spectrum corrected
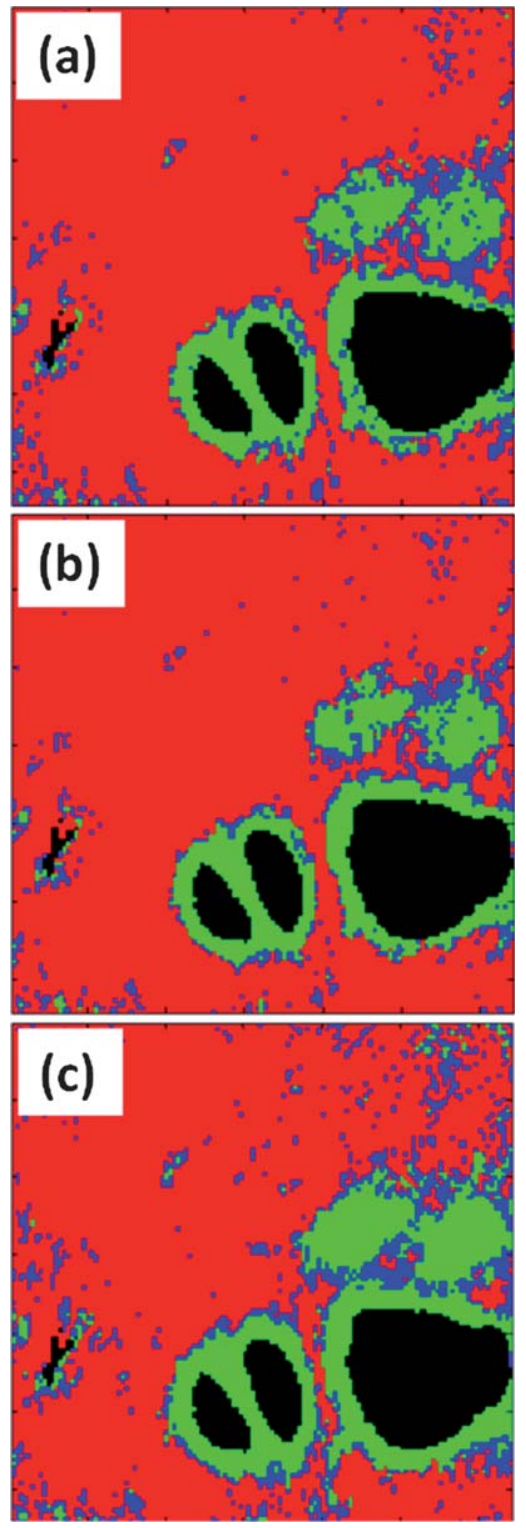

Fig. 8 Classification of patient 2 tissue, corrected using (a) reference spectrum 1 (Matrigel), (b) Reference spectrum 2 (ATR Prostate), and (c) reference spectrum 3 (ATR Renal). In each case the patient 1 tissue corrected with reference spectrum 1 was used to train the classification model. 
with the three reference spectra are identical. At this point there is essentially no influence of the reference spectrum.

\subsection{The influence of the reference spectrum on imaging}

Fig. 8 shows the ANN (same model as stated in section 3.2) classification image of patient 2 with the raw spectra having been corrected with the three reference spectra using 20 iterations shown in Fig. 6. Again it is clear that the choice of reference spectrum has very little difference on the outcome of the image analysis. The classification images are not identical, however the differences between them are relatively small. It would be expected that during any pre-processing and analysis, the choice of reference spectrum used during any use of the RMieS-EMSC should be constant, however in the eventuality that it is not, then data analysis will not be adversely affected.

\section{Conclusion}

Recent studies have shown that utilisation of the RMieS-EMSC algorithm improves data quality and subsequent analysis in both single biological cells ${ }^{12,13}$ and imaging data. ${ }^{28}$

In this paper, we have addressed concerns raised by users of the RMieS-EMSC algorithm. Using an objective method of testing, we have demonstrated an iterative improvement in classification accuracy of infrared spectra, irrespective of the reference spectrum used. Importantly, we have shown that significant improvements can be made with few iterations, especially for applications relating to clustering of simulated and real data. Furthermore, using Condor high throughput computing, we have engineered a solution for reducing computation time for correcting scattering effects from large infrared datasets. Continual development of the RMieS-EMSC algorithm is likely to further improve the study of biomedical materials, using infrared spectroscopy.

\section{References}

1 D. I. Ellis and R. Goodacre, Metabolic fingerprinting in disease diagnosis: biomedical applications of infrared and Raman spectroscopy, Analyst, 2006, 131(8), 875-885.

2 C. Matthaus, B. Bird, M. Miljkovic, T. Chernenko, M. Romeo and M. Diem, Infrared and Raman Microscopy in Cell Biology, Methods Cell Biol., 2008, 89, 275.

3 L. M. Miller and P. Dumas, From structure to cellular mechanism with infrared microspectroscopy, Curr. Opin. Struct. Biol., 2010, 20(5), 649-656.

4 N. Stone, C. Kendall, M. Isabelle, F. Bazant-Hegemark, J. Hutchings, L. Orr, J. Babrah and R. Baker, Vibrational spectroscopy: a clinical tool for cancer diagnostics, Analyst, 2009, 134(6), 1029-1045.

5 P. Bassan, H. J. Byrne, F. Bonnier, J. Lee, P. Dumas and P. Gardner, Resonant Mie scattering in infrared spectroscopy of biological materials - understanding the 'dispersion artefact', Analyst, 2009, 134(8), 1586-1593.

6 P. Bassan, H. J. Byrne, J. Lee, F. Bonnier, C. Clarke, P. Dumas, E. Gazi, M. D. Brown, N. W. Clarke and P. Gardner, Reflection contributions to the dispersion artefact in FTIR spectra of single biological cells (vol. 134, pg 1171, 2009), Analyst, 2009, 134(12), 2484-2484.

7 M. Diem, B. Mohlenhoff, M. Romeo and B. R. Woody, Mie-type scattering and non-Beer-Lambert absorption behavior of human cells in infrared microspectroscopy, Biophys. J., 2005, 88(5), 36353640 .
8 J. Lee, E. Gazi, J. Dwyer, M. D. Brown, N. W. Clarke, J. M. Nicholson and P. Gardner, Optical artefacts in transflection mode FTIR microspectroscopic images of single cells on a biological support: the effect of back-scattering into collection optics, Analyst, 2007, 132(8), 750-755.

9 citauth $>$ P. Bassan, A. Kohler, H. Martens, J. Lee, H. J. Byrne, P. Dumas, E. Gazi, M. Brown, N. Clarke and P. Gardner, Resonant Mie Scattering (RMieS) correction of infrared spectra from highly scattering biological samples, Analyst, 2010, 135(2), 268-277.

10 P. Bassan, A. Kohler, H. Martens, J. Lee, E. Jackson, N. Lockyer, P. Dumas, M. Brown, N. Clarke and P. Gardner, RMieS-EMSC correction for infrared spectra of biological cells: Extension using full Mie theory and GPU computing, J. Biophotonics, 2010, 3(8-9), 609-620.

11 A. Kohler, J. Sule-Suso, G. D. Sockalingum, M. Tobin, F. Bahrami, Y. Yang, J. Pijanka, P. Dumas, M. Cotte, D. G. van Pittius, G. Parkes and H. Martens, Estimating and correcting Mie scattering in synchrotron-based microscopic Fourier transform infrared spectra by extended multiplicative signal correction, Appl. Spectrosc., 2008, 62(3), 259-266.

12 K. R. Flower, I. Khalifa, P. Bassan, D. Demoulin, E. Jackson, N. P. Lockyer, A. T. McGown, P. Miles, L. Vaccari and P. Gardner, Synchrotron FTIR analysis of drug treated ovarian A2780 cells: an ability to differentiate cell response to different drugs?, Analyst, 2011, 136(3), 498-507.

13 C. Hughes, M. Liew, A. Sachdeva, P. Bassan, P. Dumas, C. A. Hart, M. D. Brown, N. W. Clarke and P. Gardner, SRFTIR spectroscopy of renal epithelial carcinoma side population cells displaying stem cell-like characteristics, Analyst, 2010, 135(12), 3133-3141.

14 E. Gazi, J. Dwyer, N. P. Lockyer, J. Miyan, P. Gardner, C. Hart, M. Brown and N. W. Clarke, Fixation protocols for subcellular imaging by synchrotron-based Fourier transform infrared microspectroscopy, Biopolymers, 2005, 77(1), 18-30.

15 J. Woodcock-Mitchell, R. Eichner, W. G. Nelson and T. T. Sun, Immunolocalization of keratin polypeptides in human epidermis using monoclonal antibodies, J. Cell Biol., 1982, 95(2 Pt 1), $580-8$.

$16 \mathrm{~J}$. H. Ward, Hierarchical Grouping to Optimize an Objective Function, J. Am. Stat. Assoc., 1963, 58(301), 236.

17 A. Oust, T. Moretro, C. Kirschner, J. A. Narvhus and A. Kohler, FTIR spectroscopy for identification of closely related lactobacilli, $J$. Microbiol. Methods, 2004, 59(2), 149-62.

18 P. Lasch, W. Haensch, D. Naumann and M. Diem, Imaging of colorectal adenocarcinoma using FT-IR microspectroscopy and cluster analysis, Biochimica Et Biophysica Acta, 2004, 1688(2), 17686.

19 P. Lasch, M. Diem, W. Hansch and D. Naumann, Artificial neural networks as supervised techniques for FT-IR microspectroscopic imaging, J. Chemom., 2006, 20(5), 209-220.

20 D. Thain, T. Tannenbaum and M. Livny, Distributed computing in practice: the Condor experience, Concurrency and Computation: Practice and Experience, 2005, 17(2-4), 323-356.

21 T. J. Harvey, E. Gazi, A. Henderson, R. D. Snook, N. W. Clarke, M. Brown and P. Gardner, Factors influencing the discrimination and classification of prostate cancer cell lines by FTIR microspectroscopy, Analyst, 2009, 134(6), 1083-91.

22 H. C. v. d. Hulst, Light scattering by small particles. Wiley: New York, 1957.

23 B. Bird, M. Miljkovic, M. J. Romeo, J. Smith, N. Stone, M. W. George and M. Diem, Infrared micro-spectral imaging: distinction of tissue types in axillary lymph node histology, $B M C$ Clin. Pathol., 2008, 8, 8.

24 E. Gazi, J. Dwyer, P. Gardner, A. Ghanbari-Siahkali, A. P. Wade, J. Miyan, N. P. Lockyer, J. C. Vickerman, N. W. Clarke, J. H. Shanks, L. J. Scott, C. A. Hart and M. Brown, Applications of Fourier transform infrared microspectroscopy in studies of benign prostate and prostate cancer. A pilot study, J. Pathol., 2003, 201(1), 99-108.

25 E. Gazi, J. Dwyer, N. Lockyer, P. Gardner, J. C. Vickerman, J. Miyan, C. A. Hart, M. Brown, J. H. Shanks and N. Clarke, The combined application of FTIR microspectroscopy and ToF-SIMS imaging in the study of prostate cancer, Faraday Discuss., 2004, 126, 41-59. 
26 D. C. Fernandez, R. Bhargava, S. M. Hewitt and I. W. Levin, Infrared spectroscopic imaging for histopathologic recognition, Nat. Biotechnol., 2005, 23(4), 469-474.

27 B. Bird, M. Miljkovic and M. Diem, Two step resonant Mie scattering correction of infrared micro-spectral data: human lymph node tissue, J. Biophotonics, 2010, 3(8-9), 597-608.
28 K. R. Bambery, B. R. Wood and D. McNaughton, Resonant Mie Scattering (RMieS) correction applied to FTIR images of biological tissue samples, Analyst, 2011, Submitted.

29 B. Mohlenhoff, M. Romeo, M. Diem and B. R. Woody, Mie-type scattering and non-Beer-Lambert absorption behavior of human cells in infrared microspectroscopy, Biophys. J., 2005, 88(5), 3635-3640. 